ARTICLE

\title{
DNA-assembled superconducting 3D nanoscale architectures
}

\author{
Lior Shani, ${ }^{1,7}$, Aaron N. Michelson (10) ${ }^{3,7}$, Brian Minevich ${ }^{4}$, Yafit Fleger ${ }^{2}$, Michael Stern ${ }^{5}$, Avner Shaulov ${ }^{1,2}$, \\ Yosef Yeshurun ${ }^{1,2 \otimes}$ \& Oleg Gang (1) ${ }^{3,4,6 凶}$
}

Studies of nanoscale superconducting structures have revealed various physical phenomena and led to the development of a wide range of applications. Most of these studies concentrated on one- and two-dimensional structures due to the lack of approaches for creation of fully engineered three-dimensional (3D) nanostructures. Here, we present a 'bottom-up' method to create 3D superconducting nanostructures with prescribed multiscale organization using DNA-based self-assembly methods. We assemble 3D DNA superlattices from octahedral DNA frames with incorporated nanoparticles, through connecting frames at their vertices, which result in cubic superlattices with a $48 \mathrm{~nm}$ unit cell. The superconductive superlattice is formed by converting a DNA superlattice first into highly-structured 3D silica scaffold, to turn it from a soft and liquid-environment dependent macromolecular construction into a solid structure, following by its coating with superconducting niobium ( $\mathrm{Nb}$ ). Through low-temperature electrical characterization we demonstrate that this process creates $3 \mathrm{D}$ arrays of Josephson junctions. This approach may be utilized in development of a variety of applications such as 3D Superconducting Quantum interference Devices (SQUIDs) for measurement of the magnetic field vector, highly sensitive Superconducting Quantum Interference Filters (SQIFs), and parametric amplifiers for quantum information systems.

\footnotetext{
${ }^{1}$ Institute of Superconductivity, Department of Physics, Bar-Ilan University, 5290002 Ramat-Gan, Israel. ${ }^{2}$ Bar-Ilan Institute of Nanotechnology and Advanced Materials (BINA), 5290002 Ramat-Gan, Israel. ${ }^{3}$ Department of Applied Physics and Applied Mathematics, Columbia University, New York, NY 10027, USA. ${ }^{4}$ Department of Chemical Engineering, Columbia University, New York, NY 10027, USA. ${ }^{5}$ Quantum Nanoelectronics Laboratory, Department of Physics, Bar-Ilan University, 5290002 Ramat-Gan, Israel. ${ }^{6}$ Center for Functional Nanomaterials, Brookhaven National Laboratory, Upton, NY 11973, USA. ${ }^{7}$ These authors contributed equally: Lior Shani, Aaron N. Michelson.凶email: yeshurun@mail.biu.ac.il; og2226@columbia.edu
} 
T raditional nanofabrication methods ${ }^{1}$ exploiting "top-bottom" construction techniques, e.g., e-beam lithography, have been successful in producing one-dimensional (1D) and two-dimensional (2D) superconducting nanostructures, but have shown their limitation in producing three-dimensional (3D) nanostructures. In contrast, self-assembly methods offered the ability to create 3D nano-structured materials, but they were limited in ability to create prescribed 3D architectures of superconducting materials. Recently, a self-assembly of diblock polymers in gyroid phase was shown as a way to create 3D mesoporous superconductors ${ }^{2}$. This approach evidently demonstrates an exceptional power of self-assembly in creating 3D nano-structured superconductors, however, the diversity of possible nanostructures might be limited by the phase diagram of diblock polymers.

Over the past decades, the role of DNA in constructing nanoscale structures has been increasingly recognized $^{3-8}$. In particular, DNA-based assembly methods present opportunities for creating engineered 3D nanoscale structures and integrate them with functional inorganic nano-components ${ }^{9-12}$. Recent studies demonstrate that such highly engineered object as DNA origami ${ }^{13}$, which emerged as a powerful technique for fabrication of complex shaped nano-objects ${ }^{14-18}$ and targeted nanoparticle clusters $^{19,20}$, can be utilized for assembly of 3D lattices ${ }^{21-23}$. DNA origami is a process of molecular self-folding, which involves the folding of a long single strand of DNA, aided by multiple smaller "staple" strands. These staple strands bind the long strand in predetermined places, resulting in the formation of a pre-defined nanoscale structures. DNA origami nanostructures are addressable with nanometer precision, making them suitable for building precise organization of functional materials with optical, magnetic and catalytic functions ${ }^{24-28}$. Recently, approaches for creating designed 3D superlattices from 3D DNA origami frames and nanoparticles of different kinds were demonstrated ${ }^{9}, 17$. This allowed to transfer advances from design of individual DNA constructs into a fabrication of architectured 3D nanostructured matter $17,21,22$ in which optical and chemical functions were revealed ${ }^{17}$. Parallel to the self-assembly, templating processes are needed to introduce desired material properties. For, example, DNA strands were proposed to construct the scaffold for integrated 2D molecular electronics, and to be used for patterning and other applications. Therefore, approaches for metalizing DNA and DNA origami were explored ${ }^{29-32}$, as well as mineralization of DNA into carbon ${ }^{33}$ and silicated structures ${ }^{34,35}$ was reported.

In this work, we show that the flexibility of the DNA-assembly can serve as a platform for both assembly of complex, predesigned 3D periodic nanoscale architecture, and its templating with superconducting material. The 3D DNA superlattice was assembled from DNA origami frames and functionalized by coating it with superconducting $\mathrm{Nb}$. The resulting structure-a 3D array of Josephson junctions-was confirmed by detailed microscopic structural and chemical mapping studies and magneto-transport measurements.

\section{Results}

Assembly of DNA superlattice. DNA origami octahedral frames (Fig. 1a) were designed using caDNAno software ${ }^{36}$, as previously desribed ${ }^{37}$. Briefly, each edge of the frames is composed of a sixhelix bundle (6HB) of $28.6 \mathrm{~nm}$ length ( 84 base pairs). One singlestranded $\approx 2$ nm DNA chain ("sticky end") at each end of the $6 \mathrm{HB}$ is designed to be complementary to the DNA chain of the opposing DNA origami. In order to enhance structural characterization, $10 \mathrm{~nm}$ gold nanoparticles (AuNP) were inserted into the octahedron cage (golden spheres in Fig. 1b), using a DNA shell complementary to the inner strands of the frame ${ }^{17}$. We then designed an assembly of a simple cubic superlattice of octahedra (57 $\mathrm{nm}$ unit cell in an aqueous environment) with two pairs of frames designed with specific DNA strands targeting four complementary counterparts in-plane (blue) and two counterparts out-of-plane (red), as shown in Fig. 1b. This design allows placing AuNP in every alternating layer in out-plane direction, thus forming tetragonal lattice in respect to AuNP (Supplementary Fig. S1), see S4 for the lattice, frames, and AuNP design details. The separation of in-plane and out-of-plane bonds facilitates a faster in-plane growth that yields platelet type crystals, which is beneficial for $\mathrm{Nb}$ deposition steps and electrical characterization, as we discuss below.

The formed superlattices samples are flakes of $5-10 \mu \mathrm{m}$ in length and $1-3 \mu \mathrm{m}$ in thickness. Superlattice flakes were converted from a DNA material into a solid structure by sol-gel wet chemistry, growing a layer of silica on the DNA bundles. Probing the silicated structure with small angle X-ray scattering (SAXS) reveals a simple cubic arrangement of octahedron and a tetragonal arrangement of AuNP on the superlattice shown in Fig. 1d. The process of silication resulted in a shrinking of the unit cell to $48 \times 48 \times 48 \mathrm{~nm}^{3}$ as a precursor template for $\mathrm{Nb}$ deposition. For further details please see Supplementary Information S1. The created 3D silicated structures were further imaged using a scanning electron microscopy (SEM), as shown in the representative images in the Fig. 1e, f. The electrical properties of the $\mathrm{Nb}$-coated superlattices were probed by low-temperature electrical measurements (Fig. 1g), as we discuss below.

Formation of superconducting superlattice. The silica converted superlattices were dispersed on a silicon chip and coated with about $10 \mathrm{~nm}$ thick layer of $\mathrm{Nb}$, using e-beam evaporation at room temperature. The challenge in this process is to coat the structure while maintaining the lattice architecture defined by the octahedra frames. This includes avoiding sealing the voids of the structure in order to ensure penetration of the $\mathrm{Nb}$ through the layers and allowing coating of inner layers. At the same time, it is important to prevent penetration of $\mathrm{Nb}$ all the way down to the bottom of the sample, in order to avoid shorts between the electrodes in the electrical measurements. We met these requirements by controlling the evaporation rate and the temperature of the substrate, see Supplementary Information S1 for details. Figure $2 \mathrm{a}$ shows a SEM image of the top layer of one of the $\mathrm{Nb}$-coated superlattice flakes. The $\mathrm{Nb}$ accumulate at the octahederal DNA structures, creating an array of relatively large $\mathrm{Nb}$ grains, connected by the Nb-coated sticky ends between the octahedra. The inset to Fig. $2 b$ is a schematic view of four such grains and the weak links that connect them. As apparent from the geometry, each pair of superconducting octahedron is connected by a weak link shorter than the $\mathrm{Nb}$ superconducting coherence length $(\sim 40 \mathrm{~nm})$, creating an ordered 3D array of $S-s-S$ Josephson junctions (marked with a yellow patch in the inset), where $S$ stands for the large superconducting grain and $s$ for the smaller link connecting them.

In order to determine the degree of penetration of the $\mathrm{Nb}$ after the evaporation process, the sample was thinned from both the top and bottom, using Focused Ion Beam (Helius Dual Beam, FEI) milling, to yield a $600 \mathrm{~nm}$ slab of material with 10-12 layers. The top layer of this slab, i.e., an inner layer of the superlattices, was examined by Scanning transmission electron microscopy with energy dispersive spectroscopy (STEM-EDS) at the $200 \mathrm{keV}$ FEI Talos microscope. Figure $2 \mathrm{c}$ is a STEM image of the inner layers, confirming a porous superlattice structure with AuNP tracers showing bright and with the silica-coated DNA showing a hollow 
a

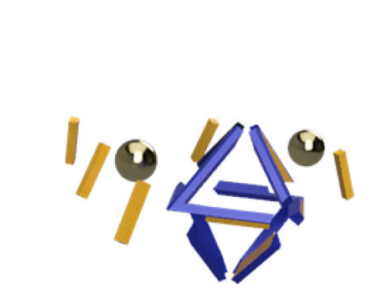

$(1)$

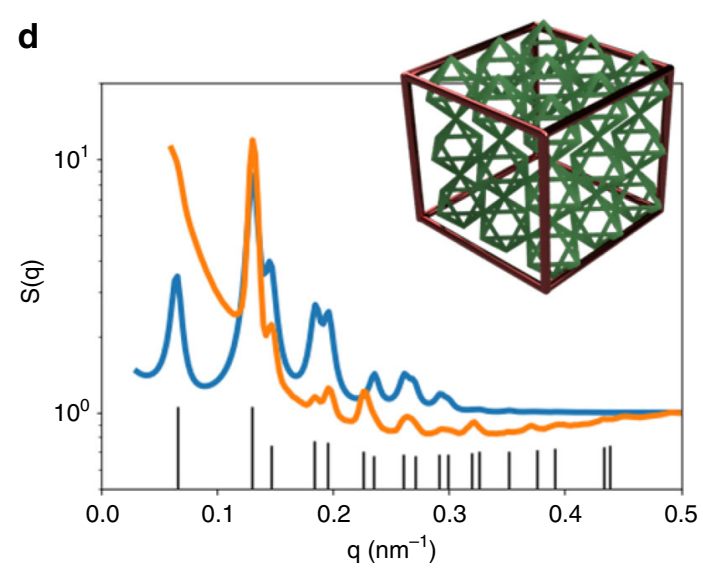

b

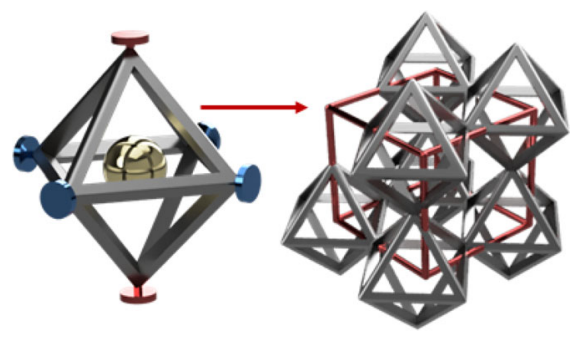

C

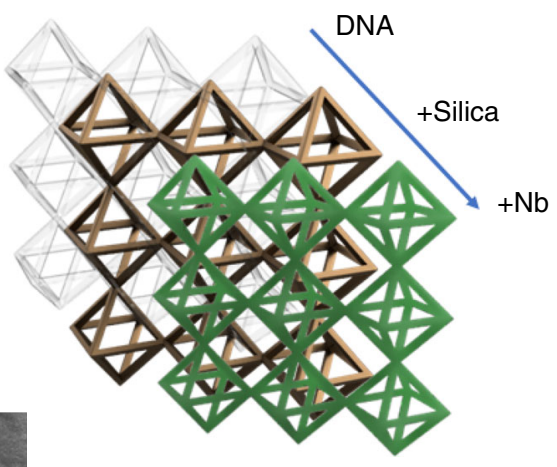

$\mathbf{9}$

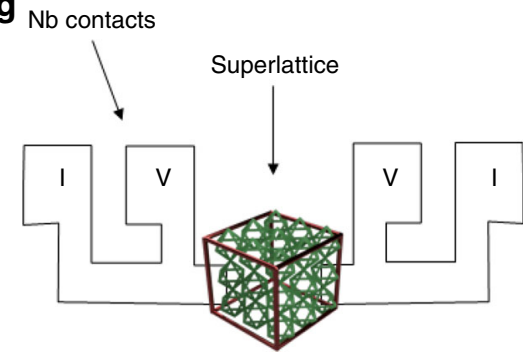

Fig. 1 Schematics of 3D superlattice assembly from octahedra DNA frames and gold nanoparticle, and its conversion into silica and superconductive structure. a DNA origami octahedral frames. b Integration of DNA frames with gold $10 \mathrm{~nm}$ nanoparticles and assembly of frames into superlattice with cubic unit cell. (AuNP used here for structural characterization and are not shown in other schematics). c Stepwise conversion process from DNA superlattices to $\mathrm{SiO}_{2}$ and to $\mathrm{Nb}$-coated structures. d Schematic of the formed simple cubic arrangement of octahedra frames. A tetragonal arrangement AuNP's is determined by SAXS: data (orange), model (blue), indexed diffraction peaks, (001), (100), (101), (111), (002), (200) etc., are shown with vertical black lines. SEM micrographs of $\mathbf{e}$ large scale and $\mathbf{f}$ close up images of fabricated silica superlattices. $\mathbf{g}$ Schematic of low-temperature electrical measurement setup for $\mathrm{Nb}$ superlattice using four-point probe.

framework. This structure was not filled in by $\mathrm{Nb}$ evaporation, and it forms a periodic array of Josephson junctions. We show in Fig. 2d, e, EDS element analysis of the outer layers of the sample, which provide information about the distribution of $\mathrm{SiO}_{2}, \mathrm{Au}$ and $\mathrm{Nb}$ in the slab. The EDS reveals the distribution of the $\mathrm{Nb}$ in the superlattice as being confined mostly to the top 3 pairs octahedra layers (note, AuNP are in every second layer, as discussed above, thus, a pair is empty octahedron and octahedron with AuNP), colored gold, red and blue from top to down respectively, with the blue further zoomed in inset to Fig. $2 \mathrm{f}$ showing the low $\mathrm{Nb}$ signal in the third layer. The AuNP signal as a reporter for superlattice position gives a representative number of layers into the superstructure with significant $\mathrm{Nb}$ deposition. By integrating column by column of superlattice EDS signal it can be determined that there was significant $\mathrm{Nb}$ on the first two layers of frames' pairs and significantly smaller $\mathrm{Nb}$ in the third pair layer, but still detectable. Thus, the superconducting lattice is formed by about six layers of octahedra frames, with the total thickness close to 290 $\mathrm{nm}$. The distribution of $\mathrm{Nb}$ is further presented in Supplementary Fig. S4 by considering the line scan of the sample with a longer dwell time, which shows only a few layers of $\mathrm{Nb}$ depositing in the top layers of the superlattice and negligible $\mathrm{Nb}$ in the interior of the superlattice (Supplementary Fig. S5).

Electrical characterization. For measuring the magnetotransport properties of the Nb-coated superlattice we used laser lithography (MLA, Heidelberg Inst.) to pattern a 4-probe setup, trapping one of the coated superlattice flakes between the voltage probes. We then isolated the flake from the $\mathrm{Nb}$ film surrounding it, using helium ion beam (ORION NanoFab, Zeiss). (We found that the commonly used gallium ion beam deteriorates the superconducting properties of the superlattice. For details, see Supplementary Information S3). The magneto-transport characteristics of the trapped superlattice were measured using Physical Properties Measurement System (PPMS, Quantum Design).

Figure 3 shows the temperature dependence of the resistance of a Nb-coated superlattice at zero field, demonstrating a superconducting transition temperature, $T_{\mathrm{c}}$, at $\sim 3.8 \mathrm{~K}$, lower than the transition temperature $(\sim 5 \mathrm{~K})$ of the reference $10 \mathrm{~nm}$ thick $\mathrm{Nb}$ film (see inset) and the transition temperature of $\sim 9.2 \mathrm{~K}$ for bulk $\mathrm{Nb}$. A reduction in $T_{\mathrm{c}}$ is expected for thin layers and wires, resulting from degradation and oxidation of the superconducting material during the fabrication process ${ }^{38-40}$.

The current-voltage $(I-V)$ characteristics of a Nb-coated superlattice (Fig. 4) were measured for temperatures between 1.9 and $3.7 \mathrm{~K}$. The corresponding low temperature curves (1.9-2.8 K) resemble the $I-V$ characteristics of a single Josephson junction, namely the voltage $V=0$ for currents up to a certain temperature-dependent critical current, $I_{c}$, indicated by the appearance of resistance, and then the voltage increases gradually, approaching a value $I R_{\mathrm{n}}$, where $R_{\mathrm{n}}$ is the normal resistance. As an example, we show in the right inset to Fig. 4 a fit of the data at the lowest temperature $(1.9 \mathrm{~K})$ to the $I-V$ characteristic of a Josephson junction ${ }^{41}$ :

$$
V=R_{\mathrm{n}}\left(I^{2}-I_{\mathrm{c}}^{2}\right)^{1 / 2}
$$

Using Eq. (1), a reasonable fit (solid line for the $1.9 \mathrm{~K}$ data in the inset) is obtained with $R_{\mathrm{n}}=200 \Omega$ and $I_{\mathrm{c}}=0.41 \mathrm{~mA}$. Notably, the fit does not take into account the small variations in the voltage that 


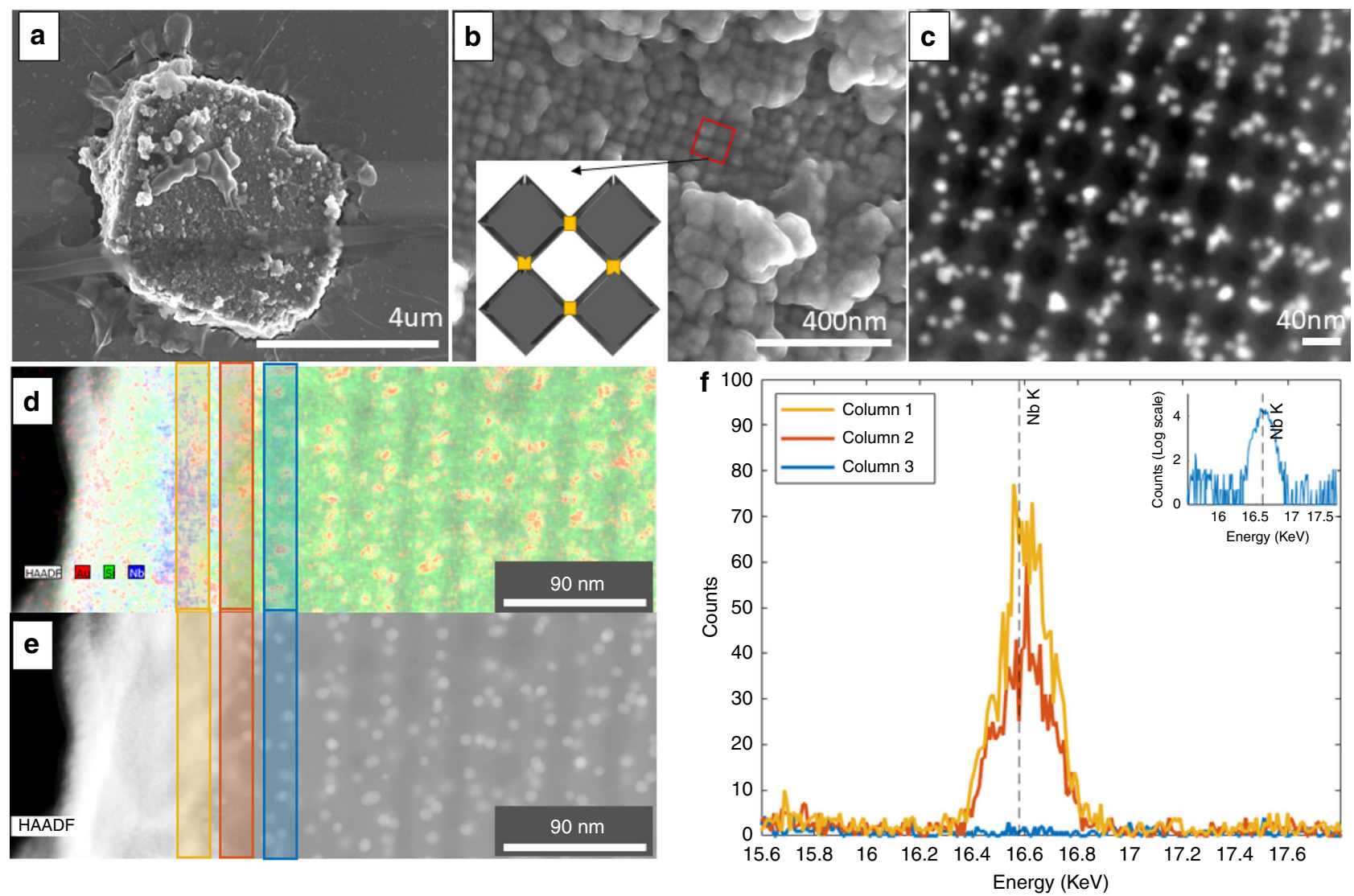

Fig. 2 Electron microscopy characterization and element analysis of Nb-coated superlattice. a SEM image of the top layer of one of the Nb-coated superlattice flakes. $\mathbf{b}$ SEM of magnified region with inset figure denoting the array arrangement of S-s-S Josephson junctions with large grains grown on the octahedron (gray diamond) with small connections formed at vertices (yellow). c HAADF image of Nb-Superlattice representative of underlying layers of surface shown in $\mathbf{b}$, AuNP appear white while the silica superlattice appears gray on a black background. $\mathbf{d}$ EDS map with column sums of first three layers (gold, red, and blue) of the Nb superlattice as indicated by the appearance of AuNP in red. e HAADF image of the same region with AuNP in white with the silica lattice in a lighter tone. $\mathbf{f}$ EDS highlighting the Nb spectrum K-edge peak at $16.58 \mathrm{KeV}$. Inset is a log scale of column 3 (blue), shown in $\mathbf{d}$, at the same energy.

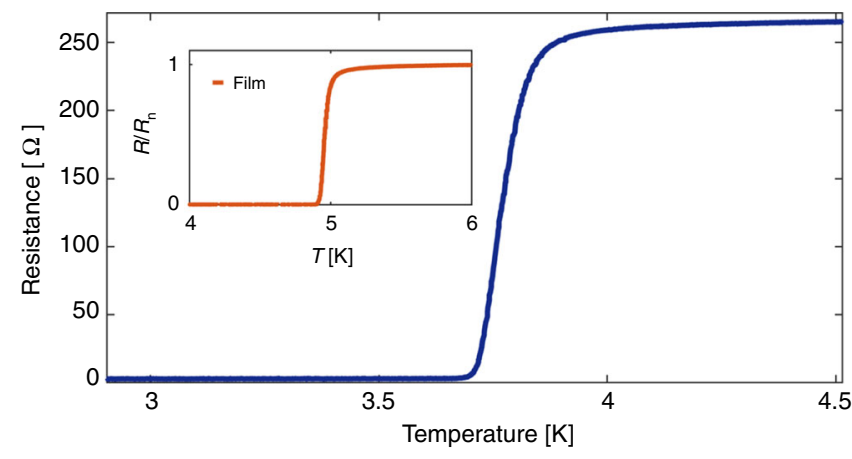

Fig. 3 Temperature dependence of the resistance of a $\mathrm{Nb}$-coated superlattice. The superconducting transition $T_{\mathrm{c}} \sim 3.8 \mathrm{~K}$ at zero field. Inset: Resistance versus temperature at zero field for the reference sample, a $10 \mathrm{~nm}$ thick $\mathrm{Nb}$ film; $T_{\mathrm{c}} \sim 5 \mathrm{~K}$.

are clearly observed in the $I-V$ characteristics. These variations imply that the array approaches the full normal state gradually, reflecting a distribution of the Josephson critical currents in the superlattice sample. The onset of a voltage at $I_{c}$ indicates that in every possible path of the current there is at least one Josephson junction, which is in the voltage state. The fact that the variations in the current are small, indicates that actually the majority of the array's junctions in most of the current paths are in the voltage

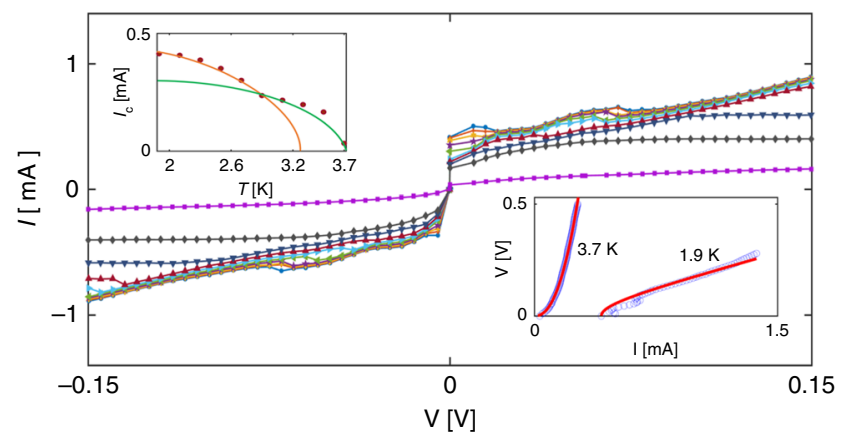

Fig. 4 Current-voltage (I-V) characteristics of a $\mathrm{Nb}$-coated superlattice. I-V characteristics for 1.9-3.7 K with $\Delta T=0.2 \mathrm{~K}$. Right inset: $V$-I characteristics (open dots) at 1.9 and $3.7 \mathrm{~K}$. Solid line for the $1.9 \mathrm{~K}$ data is a fit to Equation (1). Solid line for the $3.7 \mathrm{~K}$ data is a fit to a power law, yielding $V \propto 1^{2.3}$. Left inset: Critical current derived from the $I-V$ curves as a function of temperature. The error in each point is estimated as $2-3 \%$. Solid orange and green lines are guide to the eye, emphasizing the different behavior at high and low temperatures.

state. With increasing voltage, two competing processes determine the current; on the one hand the current follows the voltage increase and on the other hand the current drops as a result of more junctions entering the voltage state. At high enough voltage, all the 


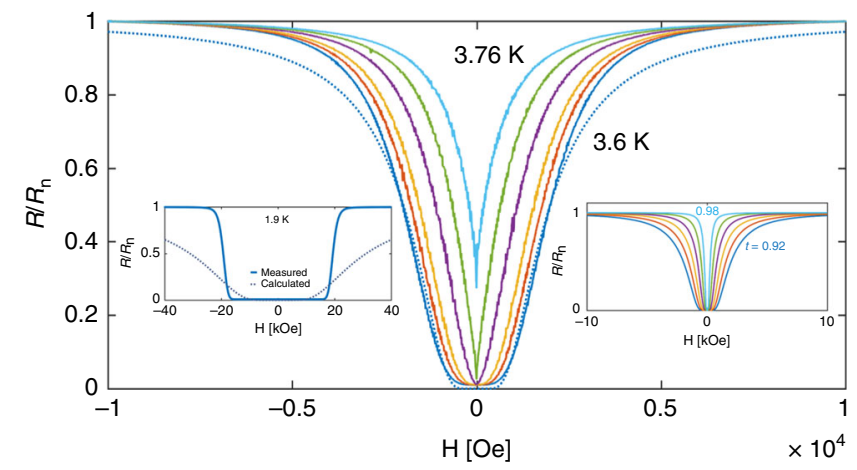

Fig. 5 Magnetoresistance of the $\mathbf{N b}$-coated superlattice as a function of the applied magnetic field. $R$ normalized to $R_{\mathrm{n}}=260 \Omega \mathrm{vs}$. $\mathrm{H}$ at temperatures $3.6,3.62,6.64,3.68,3.72$, and $3.76 \mathrm{~K}$. The dotted line is a fit to Equation (2) for $T=3.6 \mathrm{~K}$. Inset: Calculated magnetoresistance based on Equation (2) for $t=T / T_{c}=0.92$ to 0.98 .

junctions are in the voltage state and the measured voltage is that of the full normal state. Interestingly, at high temperatures $(T \geq 3.1 \mathrm{~K})$ the $I-V$ characteristics change their nature, resembling more a power-law, which characterize systems with vortex creep ${ }^{42}$. As an example of this behavior, we show in the right inset a fit of a power law (solid line) to the $3.7 \mathrm{~K}$ data, yielding $n=2.3$. The crossover between the low- and high-temperature behaviors is also observed in the critical current, $I_{\mathrm{c}}$, deduced from the $I-V$ curves. As shown in the left inset, $I_{\mathrm{c}}(T)$ exhibits discontinuity around $2.8 \mathrm{~K}$. The different behavior at high and low temperatures is presumably due to the contribution of the leads to the measured resistance. While the leads do not contribute to the resistance at low temperatures, they dominate the measured resistance at temperatures close to $T_{\mathrm{c}}$ due to vortex creep.

Qualitative different behavior at high and low temperatures is also observed in measurements of the magnetoresistance of the $\mathrm{Nb}$ coated superlattice. Figure 5 shows the resistance of this sample, normalized to its normal resistance $R_{\mathrm{n}} \sim 260 \Omega$, as a function of the applied magnetic field at temperatures close to $T_{\mathrm{c}}$. A gradual increase of the resistance is observed with the field increases. An overall behavior similar to that predicted theoretically by Tinkham ${ }^{43}$ for the case of thermally activated flux motion, namely:

$$
R / R_{\mathrm{n}}=\left(I_{0}\left[\left(\tilde{A}(1-t)^{\frac{3}{2}}\right) / 2 H\right]\right)^{-2},
$$

where $I_{0}$ is the modified Bessel function, $t=T / T_{\mathrm{c}}, \tilde{A}=80 \mathrm{~J}_{\mathrm{c} 0}$ (taking $\left.T_{\mathrm{c}}=3.8 \mathrm{~K}\right), J_{\mathrm{c} 0}$ is the critical current density at $T=0$ and $H=0$. The field $\mathrm{H}$ and the parameter $\tilde{A}$ are in units of $\mathrm{G}$, the temperature $\mathrm{T}$ in Kelvin and $J_{\mathrm{c} 0}$ is in units of $\mathrm{A} / \mathrm{cm}^{2}$. A fit of Equation (2) to the $3.6 \mathrm{~K}$ data, shown by the dotted line in the figure, yields $\tilde{A}=2.2 \times 10^{5} G$. We plot in the right inset the calculated $R / R_{\mathrm{n}}$ vs. $H$ from Equation (2) for the same temperatures shown in the main panel. The similarity between the data and the theoretical curves is apparent. The value of $\tilde{A}=2.2 \times 10^{5} \mathrm{G}$ implies $J_{\mathrm{c} 0}(0)=2.7 \times 10^{3} \mathrm{~A} / \mathrm{cm}^{2}$, a reasonable value for a granular material. However, the fitting of Equation (2) to the $\mathrm{R}(\mathrm{H})$ data fails at low temperature, as demonstrated in the left inset where we show the measured data at $1.9 \mathrm{~K}$ together with the calculated curve using $\tilde{A}=2.2 \times 10^{5} \mathrm{G}$. Clearly, the data exhibit much steeper increase from zero to $R_{\mathrm{n}}$. This behavior is more consistent with that expected from a Josephson junction in a magnetic field ${ }^{44}$. The Josephson critical current, $I_{\mathcal{c}}$, is suppressed at high fields, approaching zero in the limit of the upper critical field, $\mathrm{H}_{\mathrm{c} 2}$. As $I_{\mathrm{c}}$ drops below the measuring current, the junction enters the voltage state and gradually approaches the normal resistance as the field continues to increase.
In summary, we demonstrated, a "bottom-up" method to create 3D superconducting superlattices (with simple cubic symmetry and $48 \mathrm{~nm}$ unit cell) using self-assembly of 3D DNA frames, followed by its conversion into a periodic nanoscale $\mathrm{Nb}$ architecture. Microscopic characterization depicts that it consists of arrays of weakly linked superconducting grains, suggesting a $3 \mathrm{D}$ array of Josephson junctions. Measurements of the $I-V$ characteristics and the magnetoresistance confirm the Josephson junction behavior. This work demonstrates a conceptually different route for fabrication of complex superconducting structures that could not be prepared using conventional methods. Taking into account a tailorable platform provided by DNA-based assembly for creating engineered 3D nano-arrays ${ }^{17}$, the presented approach might be potentially utilized for a fabrication of arbitrary designed 3D superconducting materials with precise nano- and meso-scale organization.

\section{Methods}

DNA origami synthesis. DNA origami polyhedral frames were designed using caDNAno software (http://cadnano.org). Each edge of the frames is composed of a six-helix bundle (6HB). For octahedral the length of each $6 \mathrm{HB}$ is $28.6 \mathrm{~nm}$ (84 base pairs). M13mp18 DNA scaffold and DNA staple strands were mixed in a 1:5 ratio in 1x TAE buffer ( $40 \mathrm{mM}$ Tris Acetate, $1 \mathrm{mM}$ EDTA), with $12.5 \mathrm{mM} \mathrm{Mg}^{2+}$ and slowly annealed over $20 \mathrm{~h}$ from $90^{\circ} \mathrm{C}$ to room temperature over the course of $20 \mathrm{~h}$ for origami formation, overall $\mathrm{a}-0.2 \mathrm{C} / \mathrm{h}$ ramp rate.

AuNP functionalization. This method is described in [23] and reproduced here in summary. Gold NPs $(10 \mathrm{~nm})$ functionalized with citric acid were purchased from Ted Pella. NPs were modified with alkanethiol oligonucleotides by adding oligonucleotides to the aqueous NP solution at the mole ratio of 300:1 between DNA and NPs. After mixing for $2 \mathrm{~h}$, the solution was buffered at $\mathrm{pH} 7.4(10 \mathrm{mM}$ phosphate buffer). Salt $(\mathrm{NaCl})$ was added gradually to the mixture until reaching the final concentration of $0.3 \mathrm{M}$. Twelve hours later, excessive reagents were removed by centrifugation for $60 \mathrm{~min}$ at 15,700 r.c.f. and washed four times with $0.1 \mathrm{M}$ phosphate-buffered saline buffer $(0.1 \mathrm{M} \mathrm{NaCl}, 10 \mathrm{mM}$ phosphate). The nucleotides were designed to coordinate particles to the interior of the octahedron cage.

Superlattice formation. Four origami were synthesized to emphasize growth inplane. Four origami were designed with specific DNA strands targeting four complementary counterparts in plane and two counterparts out-of-plane thru a second complementary origami. The sample was annealed over five days from $50{ }^{\circ} \mathrm{C}$ to room temperature (RT) at $-0.2 \mathrm{deg} / \mathrm{h}$. The designed system is modeled in Supplementary Fig. S1a, with blue, yellow, green and red signifying DNA pairs that form the specific bond.

Silication. DNA origami superlattices were made robust by growing a layer of Silica on the DNA bundle. For conversion to inorganic silica, superlattices were centrifuged and supernatant replaced with $0.1 \times \mathrm{TAE}$ with $10 \mathrm{mM} \mathrm{Mg}$. Samples were brought into a cold room at $4^{\circ} \mathrm{C}$, incubated with (3-Aminopropyl)-triethoxysilane (APTES) for $30 \mathrm{~min}$ and then Tetraethoxysilane (TEOS) was slowly added to the lattice at a vigorous mix speed over $2 \mathrm{~h}$, incubated at $4^{\circ} \mathrm{C}$ for an additional $2 \mathrm{~h}$, then slowly brought to RT over $24 \mathrm{~h}$ in a thermomixer at $1000 \mathrm{RPM}$ from $10-20^{\circ} \mathrm{C}$ degrees. Silicated samples were drop cast to a silicon substrate. Representative images of the sample are included in Supplementary Fig. S1b-e.

Nb coating. The coating process begins with drop casting the superlattice structures on a $10 \times 10 \mathrm{~mm}^{2} \mathrm{Si}$ substrate with a native layer of oxide. The sample was dried in vacuum for $12 \mathrm{~h}$ and then inserted to "Plassys" e-beam evaporator and pumped for an additional $12 \mathrm{~h}$ to achieve optimal vacuum conditions. The sample was evaporated at a rate of $0.25 \mathrm{~nm} / \mathrm{s}$ in ambient conditions to achieve film thickness of $10 \mathrm{~nm}$ and immersed immediately in iso-propyl alcohol (IPA) to prevent oxidation. Evaporations at low temperature produced a sheet of $\mathrm{Nb}$ on top of the superlattice that did not enter the cavities.

Focused Ion Beam(FIB)/Scanning Transmission Electron Microscopy (STEM) preparation. The FIB Helios Nanolab was operated with a Gallium source to cut and mount the samples to an omniprobe grid for subsequent STEM characterization. See Supplementary Fig. S3 shows the sample in various stages of preparation.

The $200 \mathrm{KeV}$ TALOS electron microscope was then used to perform EDS mapping of the structure to determine the distribution of $\mathrm{Nb}$ on the sample with both line scans (Supplementary Fig. S4), and maps of the interior (Supplementary Fig. S5). 
Magneto-transport measurements. We fabricated two types of 4-point probe setups, using either $\mathrm{Ga}$ or He ion milling, which will be described below.

Ga ion milling. The process starts with drop casting silica superlattice on $10 \times$ $10 \mathrm{~mm}^{2} \mathrm{Si}$ chip followed by coating $\sim 10 \mathrm{~nm}$ of $\mathrm{Nb}$ using e-beam evaporator. The structure is then lifted from the film using the FIB omni-probe to a pre-patterned 4probe gold electrodes on Si chip, see Supplementary Fig. S6a. After connecting it to the electrodes using local Platinum deposition. The sample is cooled down in order to measure the resistance as a function of temperature. The measurements show a phase transition from normal to superconducting with zero electric resistance at $\approx 2 \mathrm{~K}$ that is drastically low compared to bulk Nb $\left(T_{\mathrm{c}} \approx 9.2 \mathrm{~K}\right)$, see Supplementary Fig. S6b.

He ion milling. The Nb-coated superlattice on a chip is spin coated with photoresist (AZ1518 at 4000RPM). The pattern was exposed using Mask Less Alignment (MLA) Heidelberg using $405 \mathrm{~nm}$ laser. Figure $1 \mathrm{~g}$ in the manuscript show a schematic description of the pattern.

We exposed it in a negative tone, after development we get 4-point constructs on the substrate from unexposed photoresist. To form the 4-point, it is required to remove the $\mathrm{Nb}$ layer around the protected area, this is done using $\mathrm{Cl} 2-\mathrm{BCl} 3 \mathrm{RIE}$ followed by immersing in acetone at $40{ }^{\circ} \mathrm{C}$ to remove the photoresist. The final product is a 4-point with flakes of DNA between the electrodes. To isolate the flake from its surrounding substrate we use He ion milling (Orion NanoFab, Carl Zeiss), using the He-focused ion beam with $25 \mathrm{kV}$ accelerating voltage and current of 1-2 $\mathrm{pA}$, see Supplementary Fig. S7. In this case, $T_{\mathrm{c}}=3.8 \mathrm{~K}$.

\section{Data availability}

Scattering was modeled with ScatterSim available on github.com/CFN-softbio/ ScatterSim. Other data can be made available upon reasonable request. Requests should be made to og2226@columbia.edu.

Received: 6 May 2020; Accepted: 1 October 2020;

Published online: 10 November 2020

\section{References}

1. Liddle, J. A. \& Gallatin, G. M. Nanomanufacturing: a perspective. ACS Nano 10, 2995-3014 (2016).

2. Robbins, S. W. et al. Block copolymer self-assembly-directed synthesis of mesoporous gyroidal superconductors. Sci. Adv. 2, e1501119 (2016).

3. Jones, M. R., Seeman, N. C. \& Mirkin, C. A. Programmable materials and the nature of the DNA bond. Science 347, 1260901 (2015).

4. Benson, E. et al. DNA rendering of polyhedral meshes at the nanoscale. Nature 523, 441-444 (2015).

5. Hopkins, D. S., Pekker, D., Goldbart, P. M. \& Bezryadin, A. Quantum interference device made by DNA templating of superconducting nanowires. Science 308, 1762-1765 (2005).

6. Braun, E. \& Keren, K. From DNA to transistors. Adv. Phys. 53, 441-496 (2004).

7. Stulz, E. DNA Architectonics: towards the Next Generation of Bio-inspired Materials. Chem.-A Eur. J. 18, 4456-4469 (2012)

8. Seeman, N. C. \& Gang, O. Three-dimensional molecular and nanoparticle crystallization by DNA nanotechnology. Mrs Bull. 42, 904-912 (2017).

9. Kahn, J., Minevich, B. \& Gang, O. Three-dimensional DNA-programmable nanoparticle superlattices. Curr. Opin. Biotechnol. https://doi.org/10.1016/j. copbio.2019.12.025 (2020).

10. Zhao, Z., Jacovetty, E. L., Liu, Y. \& Yan, H. Encapsulation of gold nanoparticles in a DNA origami cage. Angew. Chem. Int. Ed. Engl. 50, 2041-2044 (2011).

11. Sun, D. Z. \& Gang, O. DNA-functionalized quantum dots: fabrication, structural, and physicochemical properties. Langmuir 29, 7038-7046 (2013).

12. Zhang, Y. G., Lu, F., Yager, K. G., van der Lelie, D. \& Gang, O. A general strategy for the DNA-mediated self-assembly of functional nanoparticles into heterogeneous systems. Nat. Nanotechnol. 8, 865-872 (2013).

13. Rothemund, P. W. Folding DNA to create nanoscale shapes and patterns. Nature 440, 297 (2006).

14. Wang, P., Meyer, T. A., Pan, V., Dutta, P. K. \& Ke, Y. The beauty and utility of DNA origami. Chem 2, 359-382 (2017).

15. Tian, C. et al. Supra-nanoparticle functional assemblies through programmable stacking. ACS Nano 11, 7036-7048 (2017).

16. Gerling, T., Wagenbauer, K. F., Neuner, A. M. \& Dietz, H. Dynamic DNA devices and assemblies formed by shape-complementary, non-base pairing 3D components. Science 347, 1446-1452 (2015).

17. Tian, Y. et al. Ordered three-dimensional nanomaterials using DNAprescribed and valence-controlled material voxels. Nat. Mater. https://doi.org/ 10.1038/s41563-019-0550-x (2020).

18. Han, D. et al. DNA origami with complex curvatures in three-dimensional space. Science 332, 342-346 (2011).

19. Sun, S. et al. Valence-programmable nanoparticle architectures. Nat. Commun. 11, 1-10 (2020).
20. Roller, E.-M. et al. DNA-assembled nanoparticle rings exhibit electric and magnetic resonances at visible frequencies. Nano Lett. 15, 1368-1373 (2015).

21. Liu, W. et al. Diamond family of nanoparticle superlattices. Science 351, 582-586 (2016).

22. Tian, Y. et al. Lattice engineering through nanoparticle-DNA frameworks. Nat. Mater. 15, 654-661 (2016).

23. Zhang, T. et al. 3D DNA origami crystals. Adv. Mater. 30, e1800273 (2018)

24. Wang, P. F. et al Magnetic plasmon networks programmed by molecular selfassembly. Adv. Mater. 31, https://doi.org/10.1002/adma.201901364 (2019).

25. Zhang, $H$. et al. Polarized single-particle quantum dot emitters through programmable cluster assembly. ACS Nano 14, 1369-1378 (2020).

26. Zhao, Z. et al. Nanocaged enzymes with enhanced catalytic activity and increased stability against protease digestion. Nat. Commun. 7, 10619 (2016)

27. Urban, M. J. et al. Plasmonic toroidal metamolecules assembled by DNA origami. J. Am. Chem. Soc. 138, 5495-5498 (2016).

28. Meyer, T. A., Zhang, C., Bao, G. \& Ke, Y. Programmable assembly of iron oxide nanoparticles using DNA origami. Nano Lett. 20, 2799-2805 (2020)

29. Schreiber, R. et al. DNA origami-templated growth of arbitrarily shaped metal nanoparticles. Small 7, 1795-1799 (2011)

30. Jia, S. et al. Origami patterning with non-canonical DNA-based metallization reactions. Nat. Commun. 10, 5597 (2019).

31. Mertig, M., Colombi Ciacchi, L., Seidel, R., Pompe, W. \& De Vita, A. DNA as a selective metallization template. Nano Lett. 2, 841-844 (2002).

32. Keren, K., Berman, R. S. \& Braun, E. Patterned DNA metallization by sequencespecific localization of a reducing agent. Nano Lett. 4, 323-326 (2004).

33. Zhou, F. et al. Programmably shaped carbon nanostructure from shapeconserving carbonization of DNA. ACS Nano 10, 3069-3077 (2016)

34. Nguyen, L., Döblinger, M., Liedl, T. \& Heuer-Jungemann, A. DNA-origamitemplated silica growth by sol-gel chemistry. Angew. Chem. Int. Ed. 58, 912-916 (2019)

35. Liu, X. et al. Complex silica composite nanomaterials templated with DNA origami. Nature 559, 593-598 (2018).

36. Douglas, S. M. et al. Self-assembly of DNA into nanoscale three-dimensional shapes. Nature 459, 414-418 (2009).

37. Tian, Y. et al. Prescribed nanoparticle cluster architectures and lowdimensional arrays built using octahedral DNA origami frames. Nat Nanotechnol. 10, 637 (2015).

38. Park, S. \& Geballe, T. Tc depression in thin Nb films. Phys. B+ C. 135, 108-112 (1985).

39. Kim, H., Jamali, S. \& Rogachev, A. Superconductor-insulator transition in long moge nanowires. Phys. Rev. Lett. 109, 027002 (2012)

40. Pereiro, J., Saerbeck, T. \& Schuller, I. K. Effect of increasing disorder on superconductivity of $\mathrm{Mo} / \mathrm{Nb}$ superlattices. Superconductor Sci. Technol. 28 085001 (2015)

41. Tinkham, M. Introduction to Superconductivity. 2nd edn, 202-211, (Dover Publications Inc., 2004)

42. Yeshurun, Y., Malozemoff, A. \& Shaulov, A. Magnetic relaxation in hightemperature superconductors. Rev. Mod. Phys. 68, 911 (1996).

43. Tinkham, M. Resistive transition of high-temperature superconductors. Phys Rev. Lett. 61, 1658-1661 (1988)

44. Zhilyaev, I. Step-like dependence of the magnetoresistance of a Josephson structure. J. Surf. Investig. X-ray, Synchrotron Neutron Tech. 7, 652-654 (2013).

\section{Acknowledgements}

This work was supported by the US Department of Defense, Army Research Office, Grant W911NF-19-1-0395. The DNA design and assembly work was supported by US Department of Energy, Office of Basic Energy Sciences, Grant DE-SC0008772. This research used resources of the Center for Functional Nanomaterials, and the National Synchrotron Ligh Source II (NSLS II), supported by U.S. DOE Office of Science Facilities at Brookhaven National Laboratory under Contract No. DE-SC0012704. The authors acknowledge the Complex Materials Scattering beamline at NSLS II and the Imaging Facility of CUNY Advanced Science Research Center for instrument use, scientific and technical assistance. Y.Y. acknowledges a financial support from the Israeli Ministry of Science and Technology. L.S. acknowledges the support of Bathsheva de Rothschild Fund. M.S. acknowledges the support of the Israel Science Foundation under grants 416/15 and 1965/15. The authors acknowledge enlightening discussions with Jorge Berger and Boris Shapiro.

\section{Author contributions}

L.S., A.N.M., Y.Y., and O.G. conceived concept and designed the experiments. A.N.M prepared the DNA lattices, conducted their silication and characterized the structure. B.M. designed the DNA superlattice assembly and verified origami formation. L.S. coated the samples with $\mathrm{Nb}$ and performed the magneto-transport measurements. Y.F. conducted the ion milling and M.S. assisted in Nb coating. A.S., Y.Y., and O.G. analyzed the data. A.N.M., L.S., Y.Y., and O.G. prepared the manuscript.

\section{Competing interests}

The authors declare no competing interests. 


\section{Additional information}

Supplementary information is available for this paper at https://doi.org/10.1038/s41467020-19439-9.

Correspondence and requests for materials should be addressed to Y.Y. or O.G.

Peer review information Nature Communications thanks Jin-Woo Kim and the other, anonymous, reviewer(s) for their contribution to the peer review of this work.

Reprints and permission information is available at http://www.nature.com/reprints

Publisher's note Springer Nature remains neutral with regard to jurisdictional claims in published maps and institutional affiliations. (c) (i) Open Access This article is licensed under a Creative Commons Attribution 4.0 International License, which permits use, sharing, adaptation, distribution and reproduction in any medium or format, as long as you give appropriate credit to the original author(s) and the source, provide a link to the Creative Commons license, and indicate if changes were made. The images or other third party material in this article are included in the article's Creative Commons license, unless indicated otherwise in a credit line to the material. If material is not included in the article's Creative Commons license and your intended use is not permitted by statutory regulation or exceeds the permitted use, you will need to obtain permission directly from the copyright holder. To view a copy of this license, visit http://creativecommons.org/ licenses/by/4.0/.

(C) The Author(s) 2020 